\title{
Comparison of two new methods for the measurement of lung volumes with two standard methods
}

\author{
Ian J Cliff, Angela H Evans, Charles F A Pantin, David R Baldwin
}

\begin{abstract}
Background-The two most commonly used methods for the measurement of lung volumes are helium dilution and body plethysmography. Two methods have been developed which are both easier and less time consuming to perform. Mathematical modelling uses complex calculations from the flow-volume loop to derive total lung capacity (TLC), and the nitrogen balance technique uses nitrogen from the atmosphere to calculate lung volume in a similar way to helium dilution. This study was designed to compare the two new methods with the two standard methods.
\end{abstract}

Methods-Sixty one subjects were studied, 23 with normal lung function, 17 with restrictive airway disease, and 21 with obstructive ventilatory defects. Each subject underwent repeated measurements of TLC by each of the four methods in random order. Reproducible values were obtained for each method according to BTS/ARTP guidelines. Bland-Altman plots were constructed for comparisons between the methods and paired $t$ tests were used to assess differences in means.

Results-Bland-Altman plots showed that the differences between body plethysmography and helium dilution fell into clinically acceptable ranges (agreement limits \pm 0.91 ). The agreement between mathematical modelling or the nitrogen balance technique and helium dilution or body plethysmography was poor $( \pm 1.8-$ 3.4 1), especially for subjects with airflow obstruction.

Conclusions-Neither of the new methods agrees sufficiently with standard methods to be useful in a clinical setting.

(Thorax 1999;54:329-333)

Keywords: lung volumes; measurement; mathematical modelling; nitrogen balance

Accurate measurement of static lung volumes is an important function of the respiratory laboratory. The two most commonly used validated methods are helium dilution and body plethysmography. ${ }^{12}$ Both are time consuming and require good technical skill to produce consistent results. There is therefore a place for easier and faster methods. Two such methods have been developed recently, one based on a nitrogen gas recovery technique and one on mathematical models using meas- urements from the flow-volume curve. Both methods are commercially available as the Transflow and Spiro 232 (PK Morgan, Rainham, Kent, UK), respectively. There are no published data validating these methods and this study was therefore designed to compare each of the new methods with each of the standard methods.

\section{Methods}

SUBJECTS

Sixty one subjects were recruited from the diagnostic index of the Department of Respiratory Medicine. All gave written informed consent and the study was approved by the North Staffordshire Hospital research and ethics committee. For the purposes of the study, subjects with restrictive ventilatory defects were those who had a diagnosis consistent with such a defect and a total lung capacity (TLC) measured by helium dilution of $\leqslant 85 \%$ of the predicted value or a TLC of $\leqslant 95 \%$ and a ratio of forced expiratory volume in one second to forced vital capacity $\left(\mathrm{FEV}_{1} / \mathrm{FVC}\right)$ of $\geqslant 100 \%$ of the predicted value. Subjects with obstructive defects were required to have a consistent diagnosis and an $\mathrm{FEV}_{1} / \mathrm{FVC}$ ratio of $\leqslant 90 \%$ predicted and TLC of $\geqslant 90 \%$ predicted as measured by body plethysmography. Two subjects were excluded from the analysis because they had mixed ventilatory defects. This left 25 subjects with normal lung function, 17 with restrictive defects, and 17 with obstructive defects.

\section{RESPIRATORY FUNCTION TESTING}

Helium dilution and body plethysmography were performed strictly according to British Thoracic Society/Association of Respiratory Technicians and Physiologists (BTS/ARTP) guidelines. ${ }^{3}$ In accordance with ARTP/BTS guidelines, subjects were asked to avoid smoking for 24 hours prior to the study; consuming alcohol four hours prior to the study; vigorous exercise 30 minutes prior to the tests; wearing restrictive clothing; eating a substantial meal for two hours prior to the tests; and taking short acting bronchodilator drugs for at least four hours prior to the tests. Calibration of equipment was performed before each test. Subjects were rested for 10 minutes between each method.

We are not aware of published work on the new methods but have obtained the limited company information to provide some explanation of the physiology presented below. 
MATHEMATICAL MODELLING

Mathematical modelling ${ }^{4}$ is based on the principle that the time constant of the lung emptying depends on static lung compliance, inflow resistance, and lung volume. Lapp and Hyatt made the observation that the time constant of the lung emptying can be derived from the slope of the maximal expiratory flow-volume curve between points corresponding to 50$75 \%$ of the vital capacity. ${ }^{5}$ This flow-volume plot of forced expiration correlates well with the product of directly measured pulmonary inflow resistance and static lung compliance. These concepts are extended to the estimation of residual volume by using multiple non-linear regression equations. Variables included are the ratio of forced inspiratory flow at $50 \%$ to forced expiratory flow at 50\% lung volume $\left(\mathrm{FIF}_{50}: \mathrm{FEF}_{50}\right)$ which reflects lung compliance, the ratio of the expiratory to inspiratory areas, $\mathrm{FEV}_{1}$, and forced inspiratory vital capacity. The latter two are used as scaling factors. The specific regression equations used in the software were not available from P K Morgan. Calibration was performed prior to each subject. The sealed spirometer was checked for leaks, correct measurement confirmed with a three litre syringe, and known volumes were entered into the equipment at various speeds. Input and output volumes should not vary by more than $1.5 \%$.

The procedure consisted simply of a maximal forced expiratory and maximal forced inspiratory manoeuvre and the results were calculated automatically immediately afterwards. The results reported are recommended to be the highest $\mathrm{FEV}_{1}$ and FVC values achieved from at least three technically acceptable manoeuvres, all within $5 \%$ of each other.

\section{NITROGEN BALANCE}

In this method the lung volume is derived from the calculation of the recovery of resident nitrogen in the lung following the inhalation of a gas of known constitution. The resident nitrogen very rapidly assumes a fresh equilibrated concentration within the lung after its modification by an incoming mixture of gases. (Helium, by comparison, as a constituent of incoming gas previously not represented in the lung takes relatively longer to reach equilibrium.) Factors which favour the self-diffusion of nitrogen into a quaternary gas mixture which is itself composed largely of nitrogen include (1) a large volume of solvent gas (nitrogen) is already present in the alveolus at body temperature and hence has increased molecular velocity; (2) nitrogen is less viscous than helium; and (3) the behaviour of individual gases within quaternary gas mixtures constitutes an exception to Fick's second law of diffusion. The most important exception involves multi-component systems in which more than two species are involved in the diffusion process. The natural respired species which include nitrogen, oxygen, carbon dioxide and water, having similar molecular weights, can be treated collectively as a monocomponent gas for the purposes of diffusion. It has been shown by $\mathrm{Chang}^{6}$ that the simultane- ous introduction of two foreign gases into an air mixture will result in ternary as well as binary molecular interactions which invalidate the straightforward application of Fick's law. In addition, the introduction of a light gas (such as helium) into a relatively heavy solvent gas produces a transient pressure gradient within the heavier solvent gas. This has the effect of enhancing or promoting the translational kinetic energy (molecular motion) of the heavier gas and suppressing the motility of the lighter component. The helium component may therefore be regarded as acting as a catalyst which promotes the accelerated equilibration of the nitrogen species throughout the lung, effectively outstripping the mixing capability of the catalytic gas itself (helium). In practice, therefore, the recovery of nitrogen following a 10 second breath holding manoeuvre will reflect more faithfully the extent of the alveolar space than will the comparable distribution of the helium during the same period of equilibration. The removal of water vapour and carbon dioxide constituents from the alveolar gas of the subject breathing room air produces a nitrogen content of approximately $84 \%$. The inert gas content is determined by the subtraction of the sum of the directly measured helium, carbon monoxide, and oxygen concentrations from the whole. Following the measurement of the inspired and expired gas mixtures, the following mass balance equation applies:

$$
\begin{gathered}
\mathrm{V}_{\mathrm{A}} \mathrm{N}_{2}=\left[\mathrm{V}_{\mathrm{I}}-\mathrm{D}_{\mathrm{s}}\right] \times\left[\mathrm{F}_{\mathrm{A}} \mathrm{N}_{2}-\mathrm{F}_{\mathrm{I}} \mathrm{N}_{2}\right] / \\
{\left[\mathrm{F}_{\mathrm{A}} \mathrm{N}_{2}-\mathrm{F}_{\mathrm{E}} \mathrm{N}_{2}\right] / 0.95}
\end{gathered}
$$

where $\mathrm{V}_{\mathrm{I}}=$ volume inspired, $\mathrm{D}_{\mathrm{s}}=$ dead space volume, $\mathrm{F}_{\mathrm{A}} \mathrm{N}_{2}=$ nitrogen resident in alveoli, $\mathrm{F}_{\mathrm{I}} \mathrm{N}_{2}=$ nitrogen inspired, $\mathrm{F}_{\mathrm{E}} \mathrm{N}_{2}=$ nitrogen expired, $0.95=$ absorption of carbon dioxide prior to gas analysis, and $\mathrm{V}_{\mathrm{A}} \mathrm{N}_{2}=$ total lung capacity. The factor 0.95 represents (by convention) the allowance made, when computing lung volume, in respect of the absorption of carbon dioxide prior to gas analysis. There is no allowance made for water vapour in the calculations, which is consistent with similar methods of measuring volume-for example, alveolar volume:

$$
\text { alveolar volume }(\mathrm{VA})=\left[\mathrm{V}_{\mathrm{I}} \times \mathrm{He}_{\mathrm{I}}\right] /
$$$$
\left[\mathrm{He}_{\mathrm{E}} \times 0.95\right] \times 1.12
$$

where $0.95=$ correction for the absorption of carbon dioxide.

Calibration of the pneumotachograph was confirmed with a three litre syringe with various flow rates $( \pm 1.5 \%)$. The helium, oxygen, and carbon monoxide analysers were calibrated prior to each subject with a certified reference gas mixture.

To perform nitrogen balance the subject takes one or more tidal breaths and is instructed to breathe out fully, followed by a full inspiration and breath holding for a $10 \mathrm{sec}-$ ond period, with full expiration at the end. The analysis is then completed automatically.

\section{PROTOCOL}

All equipment was calibrated for each subject. Each of the four methods was used in random order and by the same physiologist (IJC). Randomisation was by means of computer gener- 
Table 1 Age, sex and diagnosis of study subjects

\begin{tabular}{|c|c|c|c|c|c|c|c|}
\hline Ventilatory defect & $\begin{array}{l}\text { Mean } \\
(S D) \\
\text { age }\end{array}$ & $\begin{array}{l}\text { Sex } \\
(M: F)\end{array}$ & $\begin{array}{l}\text { Pulmonary } \\
\text { fibrosis }\end{array}$ & $\begin{array}{l}\text { COPD/ } \\
\text { asthma }\end{array}$ & Sarcoidosis & Other & Normal \\
\hline Normal $(n=25)$ & $34(13)$ & $12: 13$ & 0 & 0 & 2 & & 23 \\
\hline Restrictive $(\mathrm{n}=17)$ & $61(13)$ & $9: 8$ & 10 & & 2 & 5 & \\
\hline Obstructive $(\mathrm{n}=17)$ & $54(10)$ & $10: 7$ & & 14 & 3 & & \\
\hline
\end{tabular}

ated random numbers (Microsoft Excel). Reproducible values were obtained according to BTS/ARTP guidelines. ${ }^{3}$

\section{REPEATABILITY AND QUALITY CONTROL} Mathematical modelling

The results reported were the best $\mathrm{FEV}_{1}$ and FVC values achieved from at least three technically acceptable manoeuvres. For FEV and FVC to be considered technically acceptable, the test should have been performed correctly and the chosen values should not exceed the next highest by more than $5 \%$ or 0.11 , whichever is the greater.

\section{Helium dilution}

When the subject was connected to the spirometer circuit, the helium concentration/ functional residual capacity (FRC) was recorded every 30 seconds until it was stablethat is, when two consecutive readings were within $\pm 0.025 \mathrm{l}$. At this point the final reading was noted. The subject was asked to perform a relaxed expiratory reserve volume (ERV) manoeuvre and then an inspiratory capacity (IC) manoeuvre followed by a vital capacity (VC) manoeuvre. Three ERV, IVC, and VC manoeuvres within $5 \%$ of each other were carried out in one sequence, with the largest value being reported.

\section{Body plethysmography}

A minimum of three technically acceptable traces within $5 \%$ of each other were obtained, and the mean of these reported. The line to obtain the slope of the relationship between box pressure and mouth pressure was observed to ensure that it passed through the centre of the closed loop at the point where the inspiration/expiration commenced.

\section{Nitrogen balance}

A minimum of two technically acceptable tests within $5 \%$ of each other were performed with the mean of these being reported. There was a period of at least four minutes between tests to allow washout of the test gas mixture.

Measurements of VC for each subject was within $10 \%$ for all tests. If any of the subjects was unable to fulfil the general requirements or to perform technically acceptable tests, testing was abandoned and they were omitted from the study.

ANALYSIS OF DATA

Paired $t$ tests were used to test differences between methods and the Bland and Altman method $^{7}$ was used to compare differences. Limits of agreement were calculated as twice the standard deviation of differences.

\section{Results}

STUDY POPULATION

Tables 1 and 2 show the clinical features of the subjects in each of the three groups. Table 3 shows the results of the paired $t$ tests with $95 \%$ confidence limits for differences for TLC and RV. As expected from published work, ${ }^{8}{ }^{9}$ body plethysmography gave higher values than helium dilution, particularly in the obstructive group, but confidence intervals were narrow in each group. When helium dilution or body plethysmography were compared with the nitrogen balance technique or mathematical modelling in normal subjects the results showed similar narrow 95\% confidence intervals (CI) but, in the group with restrictive airways disease, the $95 \%$ CI widened for comparisons involving nitrogen balance. In the patients with obstructive airways disease the $95 \%$ CI for all mean differences where either of the new methods was compared was more than twice

Table 2 Mean (SD) absolute and percentage predicted dynamic and static lung volumes

\begin{tabular}{|c|c|c|c|c|c|c|c|c|}
\hline \multirow[b]{2}{*}{ Ventilatory defect } & \multirow[b]{2}{*}{$F E V_{1}$} & \multirow[b]{2}{*}{$\%$} & \multirow[b]{2}{*}{$F V C$} & \multirow[b]{2}{*}{$\%$} & \multicolumn{4}{|l|}{$T L C$} \\
\hline & & & & & Helium dilution & $\%$ & $\begin{array}{l}\text { Body } \\
\text { plethysmography }\end{array}$ & $\%$ \\
\hline Normal & $3.69(0.77)$ & $107(14)$ & $4.43(0.96)$ & $108(14)$ & $6.07(1.19)$ & $104(13)$ & $6.54(1.38)$ & $112(15)$ \\
\hline Restrictive & $2.13(0.79)$ & $83(21)$ & $2.53(0.90)$ & $80(22)$ & $4.09(1.12)$ & $72(15)$ & $4.50(1.32)$ & 79 (18) \\
\hline Obstructive & $1.65(1.00)$ & $51(19)$ & $3.39(1.25)$ & $87(15)$ & $6.58(1.49)$ & 109 (19) & $7.29(1.38)$ & $122(20)$ \\
\hline
\end{tabular}

$\mathrm{FEV}_{1}=$ forced expiratory volume in one second; $\mathrm{FVC}=$ forced vital capacity; TLC = total lung capacity.

Table 3 Paired t tests for comparisons between methods of measurement of total lung capacity (TLC) and residual volume (RV). Values refer to the difference between means and the 95\% confidence interval (CI)

\begin{tabular}{|c|c|c|c|c|c|c|c|c|c|}
\hline \multirow[b]{2}{*}{ Comparison } & \multicolumn{3}{|c|}{ Normal } & \multicolumn{3}{|c|}{ Restrictive } & \multicolumn{3}{|c|}{ Obstructive } \\
\hline & Mean & $S D$ & $95 \% C I$ & Mean & $S D$ & $95 \% C I$ & Mean & $S D$ & $95 \% C I$ \\
\hline \multicolumn{10}{|l|}{ TLC } \\
\hline $\mathrm{HD}-\mathrm{MM}$ & 0.07 & 0.54 & -0.16 to 0.29 & 0.67 & 0.49 & 0.42 to 0.92 & -0.66 & 1.61 & -1.49 to 0.17 \\
\hline $\mathrm{HD}-\mathrm{NB}$ & -0.38 & 0.42 & -0.55 to -0.21 & -0.69 & 0.92 & -1.16 to -0.22 & 0.03 & 1.20 & -0.59 to 0.65 \\
\hline $\mathrm{HD}-\mathrm{BP}$ & -0.47 & 0.48 & -0.67 to -0.27 & -0.41 & 0.36 & -0.60 to -0.23 & -0.70 & 0.37 & -0.89 to -0.51 \\
\hline $\mathrm{BP}-\mathrm{MM}$ & 0.53 & 0.63 & 0.27 to 0.79 & 1.07 & 0.65 & 0.74 to 1.40 & 0.04 & 1.72 & -0.84 to 0.92 \\
\hline $\mathrm{BP}-\mathrm{NB}$ & 0.08 & 0.48 & -0.12 to 0.28 & -0.28 & 1.09 & -0.84 to 0.24 & 0.68 & 1.09 & 0.12 to 1.24 \\
\hline \multicolumn{10}{|l|}{ RV } \\
\hline $\mathrm{HD}-\mathrm{MM}$ & -0.05 & 0.44 & -0.24 to 0.13 & 0.52 & 0.51 & 0.26 to 0.78 & -1.00 & 1.57 & -1.80 to -0.19 \\
\hline $\mathrm{HD}-\mathrm{BP}$ & -0.52 & 0.54 & -0.74 to -0.30 & -0.45 & 0.41 & -0.66 to -0.24 & -0.80 & 0.42 & -1.01 to -0.58 \\
\hline $\mathrm{BP}-\mathrm{MM}$ & 0.46 & 0.63 & 0.21 to 0.73 & 0.96 & 0.70 & 0.61 to 1.33 & -0.20 & 1.74 & -0.69 to 1.10 \\
\hline
\end{tabular}

$\mathrm{HD}=$ helium dilution; $\mathrm{MM}=$ mathematical modelling; $\mathrm{NB}=$ nitrogen balance; $\mathrm{BP}=$ body plethysmography. 

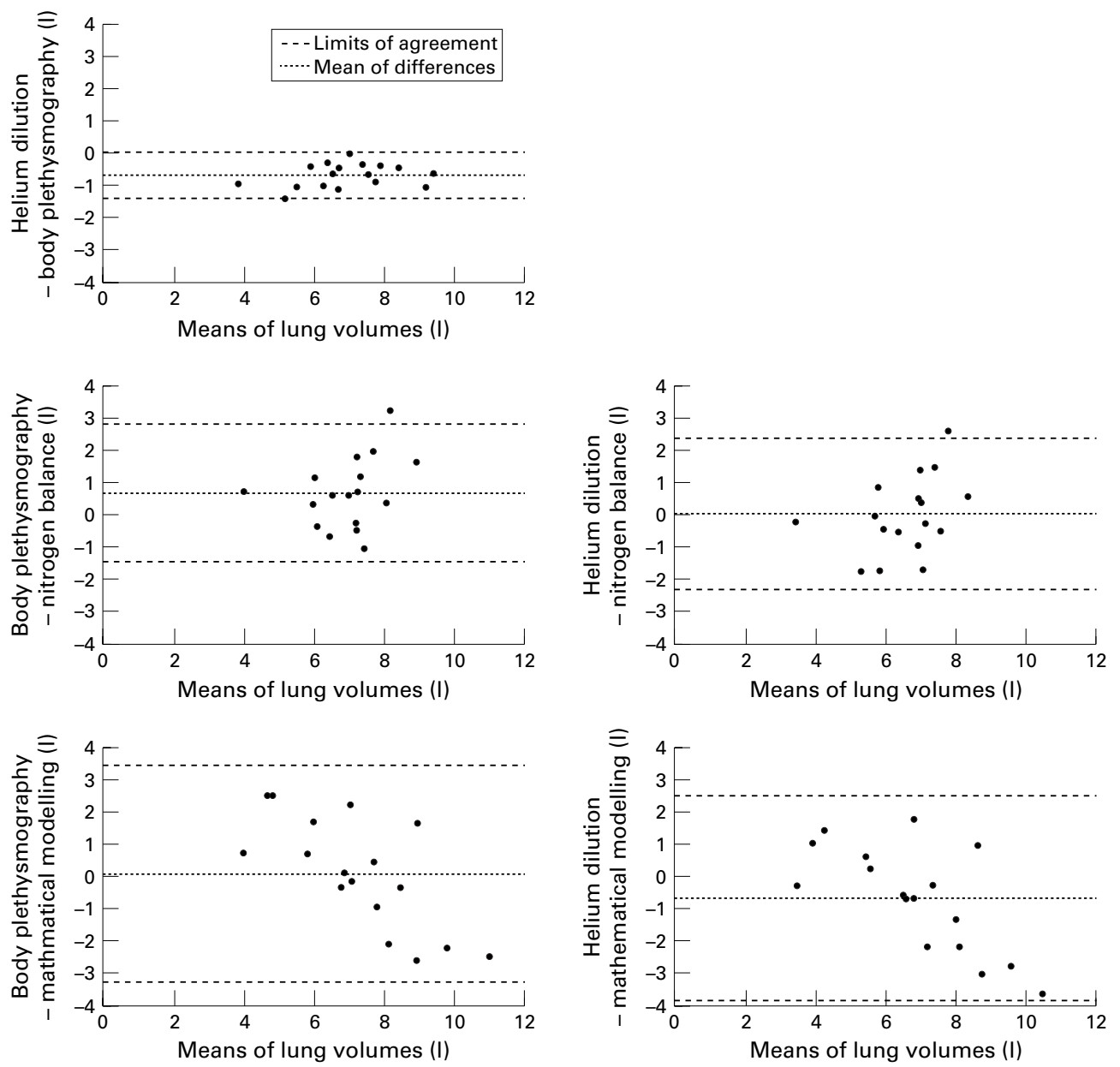

Figure 1 Bland-Altman plots for subjects with airways obstruction.

that obtained for the comparison of helium dilution with body plethysmography. BlandAltman plots were constructed for all comparisons but only those for patients with obstructive airways disease are shown in fig 1. For normal subjects there was good agreement for all comparisons. For those with restrictive airways disease the plots showed that the less good agreement for comparisons with nitrogen balance was accounted for by the presence of one outlier. The results obtained were found consistently, despite rigorous adherence to the technique described above. The subject was the only one in the study with kyphoscoliosis. The nitrogen balance technique gave a value of 6.11 with corresponding values of 2.11 and 2.21 for helium dilution and body plethysmography, respectively. Mathematical modelling tended to underestimate lung volumes compared with helium dilution and body plethysmography. Figure 1 shows the important differences in agreement for subjects with obstructive ventilatory defects. Wide limits of agreement were seen for both new methods compared with either of the standard methods. The nitrogen balance technique compared more favourably than mathematical modelling but, even with this method, the limits of agreement were around \pm 211 compared with \pm 0.91 for the standard methods. Mathematical modelling tended to overestimate larger lung volumes and underestimate small ones. Linear regression analysis showed the slope $y=-0.66 x$ +4.9 for body plethysmography versus mathematical modelling $(r=0.73, \mathrm{p}<0.001)$ and $y=$ $-0.58 x+3.4$ for helium dilution versus mathematical modelling $(r=0.7, \mathrm{p}=0.002)$.

\section{Discussion}

The techniques of mathematical modelling and nitrogen balance are both employed in equipment developed by P K Morgan, Rainham, Kent (Spiro 232 plus research software and Transflow, respectively). These systems offer other respiratory measurements (flow-volume loop in the case of Spiro 232 and transfer factor with the Transflow) as well as the measurement of TLC. Obtaining a number of lung function parameters at the same time has several potential advantages including reduction in the number of manoeuvres performed by the patient, the time and space needed, and reduced maintenance. Thus the two new methods offer an attractive promise of improved efficiency. It is therefore essential to validate these methods by comparison with those established. The present study confirms the published work comparing helium dilution with body plethysmography. ${ }^{7}$ Helium dilution estimates a lower TLC than body plethysmography, and this difference is greater with increasing airways obstruction.

Both new methods worked well for subjects with normal lung volumes but were less reliable 
for subjects with restrictive volumes and plainly misleading for those with obstructive ventilatory defects. The marked discrepancy between values obtained using nitrogen balance for the subject with kyphoscoliosis requires further investigation of other subjects with similar conditions.

The Spiro 232 gave differences in TLC in obstructive subjects of more than 31 compared with both helium dilution and body plethysmography. Subjects with obstructive airways disease and air trapping have reduced values of $\mathrm{FEV}_{1}$ and FVC. These measurements are used by the Spiro 232, and a reduction in these values may contribute to the inaccuracies found by the present study. The Transflow (nitrogen balance) did better than the Spiro 232 (mathematical modelling) but, for subjects with airways obstruction, a $95 \%$ CI of 21 is not clinically acceptable.

Bland-Altman plots of mathematical modelling versus helium dilution or body plethysmography for subjects with obstructive airway disease did show a linear relationship for which slopes were fitted. It is possible these could be used to transform data but, since the same relationship did not apply to normal subjects or those with restrictive airway disease, there would have to be some account taken of the presence of airway obstruction. Mathematical modelling is based on the most basic modelthe single compartment linear model of the lung. The exponential non-linear extension of this model is used to account for measurements made when volume approaches TLC. In patients with chronic obstructive pulmonary disease (COPD) the single compartment model may not accurately reflect the mechanical properties of the diseased lung and therefore a two compartment or even multicompartment model may be more appropriate.

The results compare better with body plethysmography, which is unexpected because nitrogen balance requires a well ventilated lung to produce accurate measurements (gas diffusion). Alveolar volume (VA) is measured while performing transfer factor, as is nitrogen balance, but both use different equations. VA compares better with helium dilution than with body plethysmography, although both underestimate lung volumes in subjects with obstructive defects. The present study did not directly compare VA with nitrogen balance but the implication is that they should not compare well. The reason why nitrogen balance compared better with body plethysmography is not fully understood, but may be related to the assumption that there is $84 \%$ nitrogen resident in the lung free of water and carbon dioxide. This may be incorrect. A further error may be produced by the indirect measurement of exhaled nitrogen where the helium, carbon monoxide, and oxygen are measured and nitrogen calculated. Further research is needed to validate assumptions by using direct measurement of nitrogen.

Transflow and Spiro 232 agree with standard methods for normal subjects. For those with restrictive defects agreement was less, though arguably still within clinically acceptable tolerances. Subjects with obstructive defects are not measured accurately, and the reasons for this are not clear. At present neither of the two new methods agrees sufficiently over the full range of disorders to be used in a clinical setting.

1 DuBois AB, Botelho SY, Bedell GN, et al. A rapid plethysmographic method for measuring thoracic gas volume: a comparison with a nitrogen washout method for measuring functional residual capacity in normal subjects. F Clin Invest 1956;35:322-6.

2 Rodenstein DO, Stanescu DC. Reassessment of lung volume measurement by helium dilution and body plethys-
mography in chronic airflow obstruction. Am Rev Respir Dis 1982;126:1040-4

3 Guidelines for the measurement of respiratory function. Respir Med 1994;88:165-94.

4 Bates JHT. Understanding lung tissue mechanics in terms of mathematical models. Monaldi Arch Chest Dis 1993,48: mather.

5 Lapp NL, Hyatt RE. Some factors affecting the relationship of maximal expiratory flow to lung volume in health and of maximal expiratory flow to lung
disease. Dis Chest $1967 ; 51: 475-81$

6 Chang HK. General concepts of molecular diffusion. In: Engel LA, Paiva M, eds. Gas mixing and distribution in the lung. Lung Biology in Health and Disease Volume 25. New York: Marcel Dekker, 1985: 1-22.

7 Bland JM, Altman DG. Statistical method for assessing agreement between two methods of clinical measurement. Lancet 1986;i:306-10.

8 Ferris BG. Recommendation standardisation procedures for pulmonary function testing. Am Rev Respir Dis 1978;118: 55-88.

9 Working Party, European Community for Coal and Steel. Standardised lung function testing: static lung volumes and capacities. Bull Eur Physiopathol Respir 1983;19(Suppl 5):11-21. 